\title{
Dosimetry of very high energy electrons (VHEE) for radiotherapy applications: using radiochromic film measurements and Monte Carlo simulations
}

\author{
A Subiel ${ }^{1}$, V Moskvin ${ }^{2}$, G H Welsh ${ }^{1}$, S Cipiccia ${ }^{1}$, D Reboredo ${ }^{1}$, \\ P Evans $^{3}$, M Partridge ${ }^{4}$, C DesRosiers ${ }^{5}$, M P Anania ${ }^{6}$, \\ A Cianchi ${ }^{7}$, A Mostacci ${ }^{6}$, E Chiadroni ${ }^{6}$, D Di Giovenale ${ }^{6}$, \\ F Villa ${ }^{6}$, R Pompili ${ }^{6}, M_{\text {Ferrario }}^{6}$, M Belleveglia ${ }^{6}$, G Di Pirro ${ }^{6}$, \\ G Gatti ${ }^{6}$, C Vaccarezza ${ }^{6}$, B Seitz ${ }^{8}, \mathbf{R}$ C Isaac $^{9}$, E Brunetti ${ }^{1}$, \\ S M Wiggins ${ }^{1}$, B Ersfeld ${ }^{1}$, M R Islam ${ }^{1}$, M S Mendonca ${ }^{5}$, \\ A Sorensen ${ }^{10}, \mathrm{M} \mathrm{Boyd}^{10}$ and D A Jaroszynski ${ }^{1}$ \\ ${ }^{1}$ Department of Physics, Scottish Universities Physics Alliance, University of \\ Strathclyde, Glasgow G4 0NG, UK \\ 2 School of Health Sciences, Purdue University, West Lafayette, Indiana, IN 47907, \\ USA \\ ${ }^{3}$ Faculty of Engineering and Physical Science, University of Surrey, Surrey GU2 \\ $7 \mathrm{XH}, \mathrm{UK}$ \\ ${ }^{4}$ Department of Oncology, University of Oxford, Oxford OX3 7DQ, UK \\ 5 Department of Radiation Oncology, Indiana University School of Medicine, \\ Indianapolis, Indiana, IN 46202, USA \\ ${ }^{6}$ Istituto Nazionale di Fisica Nucleaare, Laboratori Nazionali di Frascati, Rome, Italy \\ 7 Istituto Nazionale di Fisica Nucleaare, Roma Tor Vergata, Rome, Italy \\ 8 School of Physics and Astronomy, University of Glasgow, Glasgow G12 8QQ, UK \\ 9 Mar Athanasius College, Kothamangalam, Kerala 686 666, India \\ ${ }^{10}$ Strathclyde Institute of Pharmacy and Biomedical Sciences, University of \\ Strathclyde, Glasgow G1 1XQ, UK \\ E-mail: anna.subiel@npl.co.uk and d.a.jaroszynski@strath.ac.uk
}

Received 23 January 2014, revised 26 July 2014

Accepted for publication 1 August 2014

Published 10 September 2014

\begin{abstract}
Very high energy electrons (VHEE) in the range from $100-250 \mathrm{MeV}$ have the potential of becoming an alternative modality in radiotherapy because of their improved dosimetry properties compared with MV photons from contemporary medical linear accelerators. Due to the need for accurate dosimetry of small field size VHEE beams we have performed dose measurements using EBT2 Gafchromic ${ }^{\circledR}$ film. Calibration of the film has been
\end{abstract}


carried out for beams of two different energy ranges: $20 \mathrm{MeV}$ and $165 \mathrm{MeV}$ from conventional radio frequency linear accelerators. In addition, EBT2 film has been used for dose measurements with $135 \mathrm{MeV}$ electron beams produced by a laser-plasma wakefield accelerator. The dose response measurements and percentage depth dose profiles have been compared with calculations carried out using the general-purpose FLUKA Monte Carlo (MC) radiation transport code. The impact of induced radioactivity on film response for VHEEs has been evaluated using the MC simulations. A neutron yield of the order of $10^{-5}$ neutrons $\mathrm{cm}^{-2}$ per incident electron has been estimated and induced activity due to radionuclide production is found to have a negligible effect on total dose deposition and film response. Neutron and proton contribution to the equivalent doses are negligible for VHEE. The study demonstrates that EBT2 Gafchromic film is a reliable dosimeter that can be used for dosimetry of VHEE. The results indicate an energy-independent response of the dosimeter for $20 \mathrm{MeV}$ and $165 \mathrm{MeV}$ electron beams and has been found to be suitable for dosimetry of VHEE.

Keywords: very high energy electrons, film dosimetry, Monte Carlo, EBT2

(Some figures may appear in colour only in the online journal)

\section{Introduction}

Electron beams have been successfully used over the last 50 years as a modality in radiotherapy. Currently, clinical linear accelerators (LINACs) produce electrons with energies up to $22 \mathrm{MeV}$ (Hogstrom and Almond 2006). Such electron beams have limited application in the treatment of cancer because their dose distribution in the human body is attenuated steeply in both the longitudinal and lateral planes, which makes them unsuitable for treating deep-seated tumours. The most common treatment modality utilizes megavoltage $\mathrm{x}$-ray bremsstrahlung-generated radiation from clinical LINACs. The state of the art photon-based intensity modulated radiotherapy (IMRT) allows the radiation dose to be conformed very precisely to the three-dimensional (3D) shape of the tumour by modulating the intensity of the radiation beam. IMRT has been found to be effective in tumour treatment. However, the total integrated dose in normal tissue for IMRT treatment is a factor of two higher compared with protons (Lomax et al 1999). This aspect is particularly critical if the target volume is in the vicinity of sensitive organs.

In the past decade several studies have revived interest in radiotherapy using very high energy electron (VHEE) beams with energies exceeding $150 \mathrm{MeV}$, which allow maximum dose deposition deep in tissue. Previous theoretical studies using the PENELOPE code (DesRosiers et al 2000, Moskvin et al 2010) have shown the potential of 150-250 MeV VHEE beams for radiotherapy. The effective range of such beams can exceed $40 \mathrm{~cm}$ and, moreover, lateral scattering of high-energy electrons in tissue is sufficiently small for use in IMRT treatment of deep seated tumours (Yeboah and Sandison 2002, Fuchs et al 2009). Furthermore, the potential clinical advantage of electron beams with energies exceeding $100 \mathrm{MeV}$ have been studied for lung cancer (DesRosiers et al 2008a) and prostate cancer treatment (DesRosiers et al 2008b). These studies conclude that electron beams with energies above $100 \mathrm{MeV}$ can achieve a very good dose conformation, comparable with or even exceeding those of current photon modalities, while offering significantly better dose sparing of healthy tissue (Yeboah et al 2002). Advantages of VHEE beams include the possibility of irradiating the target volume from several different directions simultaneously, their small penumbra and high dose rates. 
Conventional VHEE LINACs based on radio frequency (RF) cavities are large devices due to the limited accelerating gradient $\left(<10 \mathrm{MV} \mathrm{m}^{-1}\right)$, imposed by electrical breakdown. Their large size (many meters in length) and high cost have limited the development of LINAC-based VHEE applications. The laser-plasma wakefield accelerator (LWFA), on the other hand, is a very compact accelerator with accelerating gradients exceeding $100 \mathrm{GV} \mathrm{m}^{-1}$, which enables energies in the $100-250 \mathrm{MeV}$ range to be obtained from mm-scale accelerators (Faure et al 2004, Geddes et al 2004, Mangles et al 2004). Electron beams from LWFAs can also have unique properties: ultra-short pulse duration (1-3fs) (Lundh et al 2011), low energy spread $(\delta \mathrm{E} / \mathrm{E}<1 \%)$ (Wiggins et al 2010), low transverse emittance $\left(\varepsilon_{n}<1\right.$ $\pi \mathrm{mm}$ mrad) and high peak current (>1 kA) (Brunetti et al 2010). Their compactness and their high quality electron beams makes them an attractive candidate for VHEE radiotherapy (Fuchs et al 2009).

The feasibility of laser-plasma accelerated electrons as a treatment modality in radiotherapy has been theoretically investigated (Glinec et al 2006) and, despite a number of simulation studies of LWFA electrons, very few experiments have been carried out. Richter (Richter et al 2011) presented dosimetry studies of low energy LWFA electrons (energy ranges corresponding to clinical LINACs). Lundh (Lundh et al 2012) conducted a study of $120 \mathrm{MeV}$ LWFA electron beams. However, no absolute dosimetric measurements have been published. Absolute dosimetry measurements for high energy electron beams are extremely important for radiobiology experiments because knowledge of the dose delivered is an essential part of evaluating radiobiological outcome. Absolute dosimetry presents a challenge for small field size beams due to high level of non-equilibrium dose deposition caused by scattering of primary particles from the small beam (Das et al 2008).

Gafchromic film is a common dosimetry tool for external beam radiotherapy and brachytherapy (Devic 2011). It has a high spatial resolution, tissue equivalence and has been successfully applied for a wide range of radiotherapy modalities.

In this paper we present dosimetric measurements using EBT2 Gafchromic film for a wide range of electron beam energies. Measurements have been made using three different accelerators: a $20 \mathrm{MeV}$ clinical LINAC, a $165 \mathrm{MeV}$ conventional linear accelerator and a $135 \mathrm{MeV}$ laser-plasma wakefield accelerator. The measurements have been compared with Monte Carlo simulations using the FLUKA general purpose code, which has been widely validated and benchmarked with experimental data (Ferrari et al 2005, Battistoni et al 2007, Parodi et al 2007, Randeniya et al 2009, Robert et al 2013).

The potential risks of VHEE radiotherapy due to secondary neutron photo-production has been raised by Bortfeld in 2004 (Papiez et al 2004). Above an energy threshold, which varies from $10-19 \mathrm{MeV}$ for light nuclei and from $4-6 \mathrm{MeV}$ for heavy nuclei (IAEA 1979), neutron production occurs in all materials arising from the electron or bremsstrahlung beam. Therefore, the application of very high energy beams requires careful consideration of the neutron generation and induced radioactivity from the standpoint of additional dose delivered and radiation protection. These aspects have been extensively described in the literature (Barbier 1969, Swanson 1978, NCRP 2003). The main channel of neutron production for VHEEs are $(\gamma, n),(\gamma, p),(\gamma, 2 n)$ and $(\gamma, p n)$ reactions. To address the concern raised by Bortfeld, the neutron yield generated by VHEEs has been calculated. To assess radiation safety, we have used MC simulations to investigate activation and the equivalent doses after irradiation with VHEEs. This also enables any additional dose deposited in the EBT2 film due to induced activity to be evaluated. 


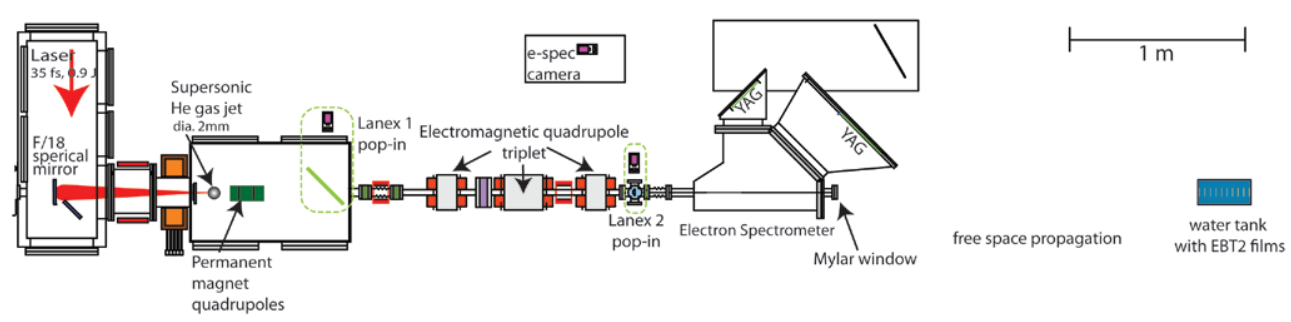

Figure 1. A schematic of the ALPHA-X laser-plasma wakefield accelerator, showing the positioning of the gas jet accelerator relative to the key detection systems and dosimetry measurements setup.

\section{Materials and methods}

\subsection{Irradiation setup}

2.1.1. ALPHA-X laboratory. The ALPHA-X laser-wakefield accelerator beam line (figure 1) in the Terahertz to Optical Pulse Source (TOPS) facility (Jaroszynski et al 2000) at the University of Strathclyde has been used to conduct experiments using LWFA VHEE beams. High power Ti:sapphire laser pulses (wavelength $\lambda=800 \mathrm{~nm}$, duration $=35 \mathrm{fs}$, energy $=0.9 \mathrm{~J}$, peak intensity $I=2 \times 10^{18} \mathrm{Wcm}^{-2}$ ) are focused with an $\mathrm{F} / 18$ spherical mirror to a spot size of $40 \mu \mathrm{m}$ (1/ $e^{2}$ diameter) on a $2 \mathrm{~mm}$ diameter pulsed supersonic helium gas jet, producing a $10 \mu \mathrm{m}$ diameter relativistic plasma channel with a density of $\sim 1.3 \times 10^{19} \mathrm{~cm}^{-3}$. High energy electron beams from the laser-plasma wakefield accelerator are imaged using a series of insertable Lanex (KODAK) phosphor screens positioned along the beam line axis.

A $22 \mathrm{~cm}$ long water phantom $\left(15 \times 30 \times 22 \mathrm{~cm}^{3}\right)$ is placed $185 \mathrm{~cm}$ after a $50 \mu \mathrm{m}$ thick Mylar vacuum window. A set of 10 EBT2 Gafchromic film sheets are equi-spaced every $2 \mathrm{~cm}$ along the beam axis in the water tank. Characterization of the electron energy spectra is carried out using a magnetic dipole imaging electron spectrometer over the range 80-240 MeV. Electron spectra are observed on scintillating Ce:YAG crystals positioned in the focal plane of the spectrometer and the image is captured on a 12-bit CCD camera. Charge recorded using Fuji BAS-SR image plates (Paterson et al 2008) is on average between 5 and $10 \mathrm{pC} /$ shot. Typical rms bunch lengths of the laser-plasma accelerated electron bunches are in the range of 1-10 fs (Shanks 2012). To estimate the charge density during the experiment, a bunch length of $5 \mathrm{fs}$ has been assumed because of dispersion arising from the beam energy spread, which gives a peak current of the order of $1 \mathrm{kA}$ at the entrance to the phantom, delivering $\sim 12 \mathrm{mGy} /$ pulse at $1.8 \mathrm{~cm}$ depth. The accelerator pulse repetition rate is restricted to $0.33 \mathrm{~Hz}$ for the dosimetry measurements. Following optimal collimation and focusing of the beam using permanent and electromagnetic quadrupole magnets, the mean transverse crosssectional diameter of the Gaussian beam at the entrance of the water tank is $1.6 \mathrm{~cm}$ full-width at half-maximum (FWHM).

2.1.2. Royal surrey hospital LINAC. Irradiation with $20 \mathrm{MeV}$ electron beams on a Varian Clinac iX (Varian) linear accelerator (Varian Medical Systems, Inc., Palo Alto, CA) has been carried out at the Royal Surrey County Hospital. This LINAC operates at $180 \mathrm{~Hz}$ pulse repetition rate, delivering $400 \mathrm{cGy} \mathrm{min}^{-1}$ at $d_{\max }$ (that corresponds to $3.7 \mathrm{mGy} / \mathrm{pulse}$ ) and produces microsecond duration nanocoulomb electron bunches at milliampere average current in a macro-pulse. 
2.1.2.1. Phantom and irradiation procedure for the surrey hospital LINAC. For exposure to the $20 \mathrm{MeV}$ electron beam, the films are placed in a standard grade solid water phantom (Gammex, Middleton, WI) composed of $20 \times 20 \mathrm{~cm}^{2}$ slabs with $5 \mathrm{~cm}$ of build-up material above the film and $15 \mathrm{~cm}$ under the film, to provide adequate backscatter. The individual films are positioned at a depth corresponding to $95 \%$ of the maximum dose for the $20 \mathrm{MeV}$ electron beam. The source-to-surface distance (SSD) is set to $100 \mathrm{~cm}$, as commonly used in radiation therapy with the $\mathrm{MeV}$ electron beams. Exposure of the films used for dose calibration is carried out using a $10 \times 10 \mathrm{~cm}^{2}$ field size, and the film is positioned perpendicularly to the axis of the beam. Calibration films are produced for five discrete exposures for doses ranging from $10 \mathrm{cGy}-100 \mathrm{cGy}$. One film is used for each dose level. Prior to irradiation, the output beam is characterized using an N34001 Roos ${ }^{\circledR}$ plane-parallel ion chamber (PTW, Freiburg, Germany) calibrated by the National Physical Laboratory (Teddington, UK).

2.1.3. INFN SPARC LAB. The SPARC (Sources for Plasma Accelerators and Radiation Compton with Lasers and Beams) LINAC test bench beamline (SPARC) has been used to perform reference dosimetry measurements for VHEE beams.

The SPARC photoinjector consists of a 1.6-cell S-band RF gun of the BNL/UCLA/SLAC type (Palmer 1998) with a $\mathrm{Cu}$ photocathode (peak electric fields of $120-140 \mathrm{MV} \mathrm{m}^{-1}$ ) and produces a $5.6 \mathrm{MeV}$ electron injection beam. Three S-band travelling wave accelerating sections raise the energy to approximately $170 \mathrm{MeV}$ (Alesini et al 2003). The transport system consists of 8 electromagnetic quadrupoles, which allow tuning of the beam properties at the target. All dosimetry measurements are performed in a $30 \times 30 \times 30 \mathrm{~cm}^{3}$ water phantom placed $41 \mathrm{~cm}$ after a $3 \mathrm{~mm}$ thick Perspex window. The energy of the SPARC electron beam for the experiment is set to $165 \mathrm{MeV}$, and the rms bunch length duration is 0.87 ps with an average charge of $60 \mathrm{pC} /$ shot, giving an electron peak current of $\sim 100 \mathrm{~A}$ and delivering $\sim 190 \mathrm{mGy} /$ pulse at the depth of $1.3 \mathrm{~cm}$. The FWHM transverse beam diameter is $0.8 \mathrm{~cm}$ at the entrance to the water tank.

2.1.3.1. Irradiation procedure of calibration films in SPARC LINAC. For exposure to a $165 \mathrm{MeV}$ electron beam from the SPARC LINAC, the films are placed in a water phantom at a depth of $2.8 \mathrm{~cm}$ from the front wall. EBT2 films are exposed to 6 discrete dose levels that correspond to different number of delivered pulses.

\subsection{Radiochromic EBT2 films}

EBT2 Gafchromic ${ }^{\circledR}$ film (Ashland 2013b) is a common secondary dosimeter used in clinical radiation therapy. The properties and sensitivity of EBT2 film have been well documented for a wide range of energies for different radiation sources used in radiotherapy (Andres et al 2010, Arjomandy et al 2010, Butson et al 2010, Lindsay et al 2010, Arjomandy et al 2012, Reinhardt et al 2012, Ashland 2013b). The MC calculated absorbed-dose energy dependence has been found to be constant for monoenergetic photon beams in the energy range from $100 \mathrm{kV}-18 \mathrm{MV}$ (Sutherland and Rogers 2010). Data provided by the manufacturer indicates that the film sensitivity may be as much as $10 \%$ higher for $\mathrm{kV} \mathrm{x}$-rays compared with MV x-rays (Ashland 2013a). The energy dependence of EBT2 film (apart from $\mathrm{kV}$ x-ray beams) has been found to be relatively small within measurement uncertainties $(1 \sigma=4.5 \%)$ for all radiotherapy energies and modalities (photon, electron and proton beams) (Arjomandy et al 2010). These energies for electron beams are substantially lower than those studied here. 


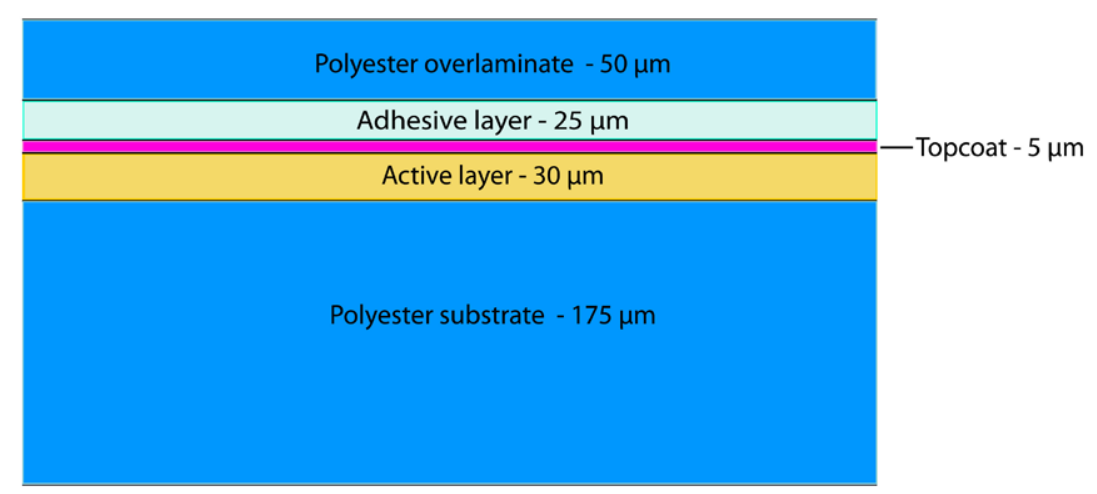

Figure 2. Configuration of Gafchromic ${ }^{\circledR}$ EBT2 dosimetry film (reproduced with permission from Ashland 2013a).

2.2.1. Film irradiation, digitising and handling procedure. The films used in the study are Gafchromic ${ }^{\circledR}$ EBT2, with sheet dimensions of $20.3 \times 25.4 \mathrm{~cm}^{2}$. They are handled according to the procedures described in the AAPM task group 55 report (Niroomand-Rad et al 1998). After irradiation, exposed as well as unexposed (for background correction) film pieces are stored together in black envelopes at room temperature to minimize exposure to light.

The films are scanned and digitized with an Epson Expression 10000XL Pro flat-bed colour scanner. The scanner is fitted with a transparency adapter and the images are acquired in transmission mode. RGB positive images are collected with 16 bit depth resolution per colour channel and a spatial resolution of $72 \mathrm{dpi}$. Software settings are chosen to disable all colour correction options and thereby produce raw scanner data without photographic enhancements. To account for the post-exposure changes all calibration films are scanned $24 \pm 2 \mathrm{~h}$ after exposure. Under these conditions errors due to time-after-exposure differences can be neglected (Ali et al 2002). It is well known that the response of EBT2 radiochromic film is sensitive to its orientation on the scanner (Paelinck et al 2007). Therefore, the film orientation in each image is recorded. The scanner response of EBT2 film is also sensitive to the position of the film on the scanner relative to the scan axis (Chung et al 2010), therefore a plastic template is used to position films in a reproducible central location in the middle of the scan window, where no correction of scan field uniformity is required. Ten preview scans are taken before the start of film scanning to allow the scanner temperature to stabilize and, thus, prevent any temperature-dependent response effects. Each film is scanned three times post-irradiation and the average of three scans is used for analysis. For film analysis, raw pixel values from the red colour channel $\left(P V_{R}\right)$ are converted into net optical density value (netOD) employing following formula:

$$
\text { netOD }=\mathrm{OD}^{\exp }-\mathrm{OD}^{\mathrm{unexp}}=-\log \left(\frac{\mathrm{PV}_{R}^{\exp }}{\mathrm{PV}_{R}^{\mathrm{unexp}}}\right),
$$

where $\mathrm{OD}^{\exp }, \mathrm{OD}^{\text {unexp}}, \mathrm{PV}^{\exp }$ and $\mathrm{PV}^{\text {unexp }}$ are the measured optical densities and pixel values of exposed and unexposed film, respectively.

2.2.1.1. Image processing. The images of the scanned films are imported into an in-house routine written in ImageJ (National Institutes of Health, Bethesda, MD) that extracts the red component of the RGB scanned image and determines the netOD of the irradiated film patches. 
Table 1. Configuration and atomic composition of Gafchromic ${ }^{\circledR}$ EBT2 film used for MC calculations.

\begin{tabular}{lcllllllllll}
\hline & \multirow{2}{*}{$\begin{array}{l}\text { Thickness } \\
{[\mu \mathrm{m}]}\end{array}$} & $\begin{array}{l}\text { Density } \\
{\left[\mathrm{g} \mathrm{cm}^{-2}\right]}\end{array}$ & \multicolumn{1}{c}{ H } & $\mathrm{Li}$ & $\mathrm{C}$ & $\mathrm{N}$ & $\mathrm{O}$ & $\mathrm{Cl}$ & $\mathrm{K}$ & $\mathrm{Br}$ \\
\hline Polyester film base & 50 & 1.35 & 36.4 & 0.0 & 45.5 & 0.0 & 18.2 & 0.0 & 0.0 & 0.0 \\
Adhesive & 25 & 1.2 & 57.1 & 0.0 & 33.3 & 0.0 & 9.5 & 0.0 & 0.0 & 0.0 \\
Topcoat & 5 & 1.2 & 56.9 & 0.9 & 25.7 & 0.0 & 15.6 & 0.9 & 0.0 & 0.0 \\
Active layer & 30 & 1.2 & 58.3 & 0.8 & 29.6 & 0.1 & 10.7 & 0.3 & 0.1 & 0.1 \\
Polyester film base & 175 & 1.35 & 36.4 & 0.0 & 45.5 & 0.1 & 18.2 & 0.0 & 0.0 & 0.0 \\
Overall composition & & & 40.85 & 0.10 & 42.37 & 0.01 & 16.59 & 0.04 & 0.01 & 0.01 \\
\hline
\end{tabular}

\subsection{Monte Carlo simulation}

The multi-purpose Monte Carlo radiation transport code FLUKA 2011.2b.4 has been used to simulate dose deposition of VHEE beams in the active layer of the EBT2 film for both ALPHA-X and SPARC beamlines. The dosimeter is modelled as a $5 \times 5 \mathrm{~cm}^{2}$ sheet composed of five layers: a polyester over-laminate, an adhesive layer, a topcoat, the active layer and a polyester substrate. Schematic diagrams of these structures are shown in figure 2 and the composition, thickness and density of the layers can be found in table 1 . The film is embedded in a $30 \times 30 \times 30 \mathrm{~cm}^{3}$ water phantom surrounded by $6 \mathrm{~mm}$ thick poly-methyl methacrylate (PMMA) walls.

Each accelerator beam has been modelled separately. The source for the SPARC beamline is a Gaussian beam with $0.43 \times 0.35 \mathrm{~cm}^{2} \mathrm{FWHM}$ size, with $15 \mathrm{mrad}$ full angle divergence. The average energy for a bunch in the SPARC beamline is set to $165 \mathrm{MeV}$, with a $0.5 \%$ energy spread approximated by a Gaussian distribution. To calculate the dose deposited in the calibration films the active layer of EBT2 film positioned at depth of $2.8 \mathrm{~cm}$ from the front wall of the water tank has been scored.

As the ALPHA-X LWFA VHEE beam exhibits shot-to-shot variations in lateral beam profile, the field shape and size is averaged over the number of shots. This profile is well approximated by a Gaussian with $0.75 \mathrm{~cm}$ by $0.7 \mathrm{~cm}$ FWHM field size and $8 \mathrm{mrad}$ divergence. User written FLUKA SOURCE subroutine (Ferrari et al 2005) is used to sample energy from experimental cumulative energy distribution curve over hundreds of shots.

The dose is scored in the active layer of the EBT2 film. The induced radioactivity is computed using RESNUCLEi cards (Ferrari et al 2005) with different time points to determine activity for various post irradiation times. Ambient dose equivalent $\mathrm{H}^{*}(10)$ from residual activity produced in the film by an electron beam is calculated using ICRP 74 data AMB74 (Pelliccioni 2000). AMB74 model adopts ICRP 74 (ICRP 1996, Pelliccioni 2000) data and contains conversion coefficients for protons, neutrons, charged pions, muons, photons and electrons.

The set of parameters PRECISIO (Ferrari et al 2005) are used to configure the physical model for the simulations. In these simulations electron/positron and photon production thresholds are set to $10 \mathrm{keV}$. The LAM-BIAS (Ferrari et al 2005) utility is applied for the photonuclear reaction simulation with the $\lambda$ (Ferrari et al 2005), inelastic setting coefficient, of 0.001. In the simulation for VHEE LINAC, 94 pulses with a duration of $0.8 \mathrm{ps}$ contain $3.3 \times 10^{8}$ e/pulse. $1 \times 10^{7}$ initial electrons trajectories are used for the calculation.

Neutron fluence inside and around the target (water phantom) is scored using the USRBIN (Ferrari et al 2005) scoring card. Because of the smaller photo nuclear cross-sections for electromagnetic interactions with atoms and electrons, the interaction length for nuclear inelastic interactions of photons is reduced in the water, the walls of the PMMA tank and all layers of EBT2 films by a factor of 1000 using the LAM-BIAS card for electron energies. 


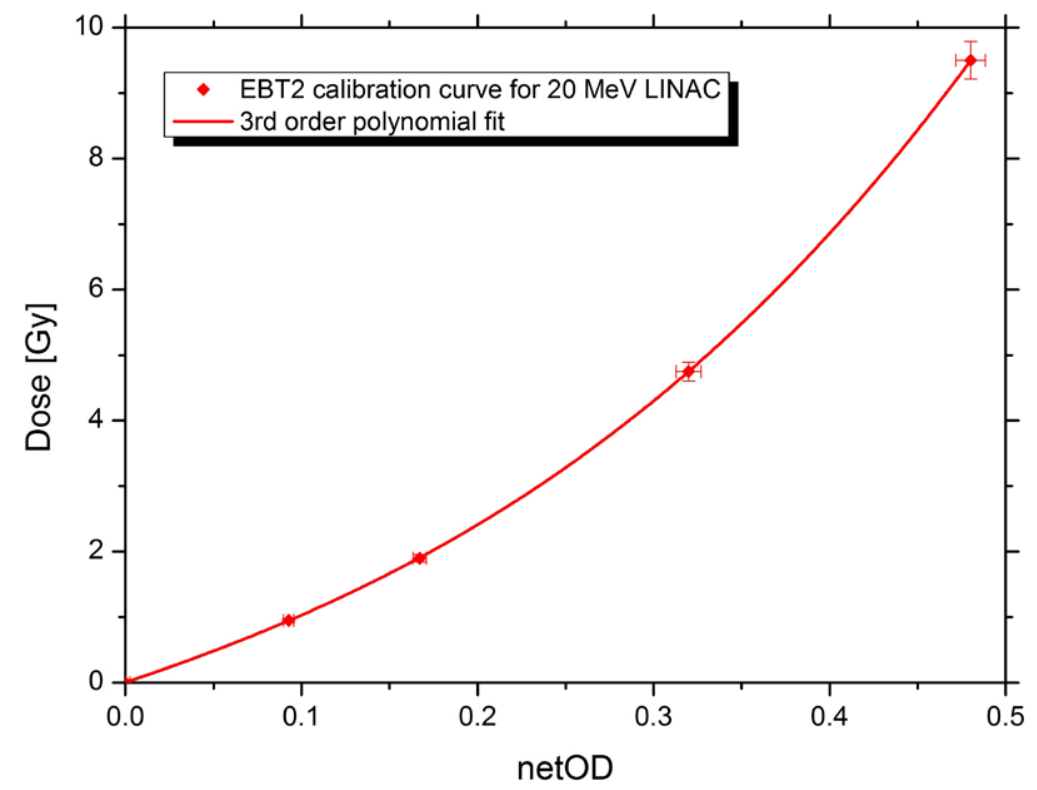

Figure 3. EBT2 film calibration curve (lot\#: A08301204) used in this study.

Table 2. Fit parameters of dose response curve. The fitting equation is: $D($ netOD $)=A_{0}+A_{1} \cdot \operatorname{netOD}+A_{2} \cdot(\text { netOD })^{2}+A_{3} \cdot(\text { netOD })^{3}$.

\begin{tabular}{lllll}
\hline \multicolumn{4}{c}{ Fit parameters } & $\Delta \mathrm{D}_{\text {tot }}[\%]$ \\
\hline $\mathrm{A}_{0}[\mathrm{~Gy}]$ & $\mathrm{A}_{1}[\mathrm{~Gy}]$ & $\mathrm{A}_{2}[\mathrm{~Gy}]$ & $\mathrm{A}_{3}[\mathrm{~Gy}]$ & 4.2 \\
$0.0014 \pm 0.0079$ & $9.05 \pm 0.16$ & $9.57 \pm 0.88$ & $26.60 \pm 1.23$ & \\
\hline
\end{tabular}

\section{Results}

\subsection{EBT2 film calibration with $20 \mathrm{MeV}$ electron beam}

The measured dose response for EBT2 film (lot\#: A08301204) irradiated with the $20 \mathrm{MeV}$ Varian iX C LINAC is shown in figure 3, where dose (in Gy) is plotted against netOD. The response curve is fitted with a third order polynomial. Fit parameters are given in table 2 . Uncertainties in the dose results in uncertainties of the netOD measurement in addition to the parameters determined during film calibration (Devic et al 2004). Dose uncertainties (Bouchard et al 2009) for lot\# A08301204 related to fit parameters are $3.75 \%$ at $10 \mathrm{~Gy}$ dose level for the calibration fit used. Accounting for additional uncertainty in the measured netOD, the combined standard uncertainty relative to the fitted dose in percentage has been determined according to the Joint Committee for Guides in Metrology (JCGM 2008) at various dose levels; for $10 \mathrm{cGy}$ and $100 \mathrm{cGy}$ it is $3.6 \%$ and $4.2 \%$, respectively. To avoid errors due to self-development of $O D$, the wait-time interval for reading $O D$ (postexposure) of all the films in the experiments is the same as the wait-time interval used to generate the calibration curve, i.e. $24 \pm 2 \mathrm{~h}$.

Two different batches of EBT2 films have been used during the experiment: lot\# A08301204 for $20 \mathrm{MeV}$ LINAC and INFN LINAC, and lot\# A04181101 for the ALPHA-X laser-plasma accelerated electron beam. For the investigated dose levels, the batch-to-batch film response varies due to small variations in film composition. We have quantified the systematic 


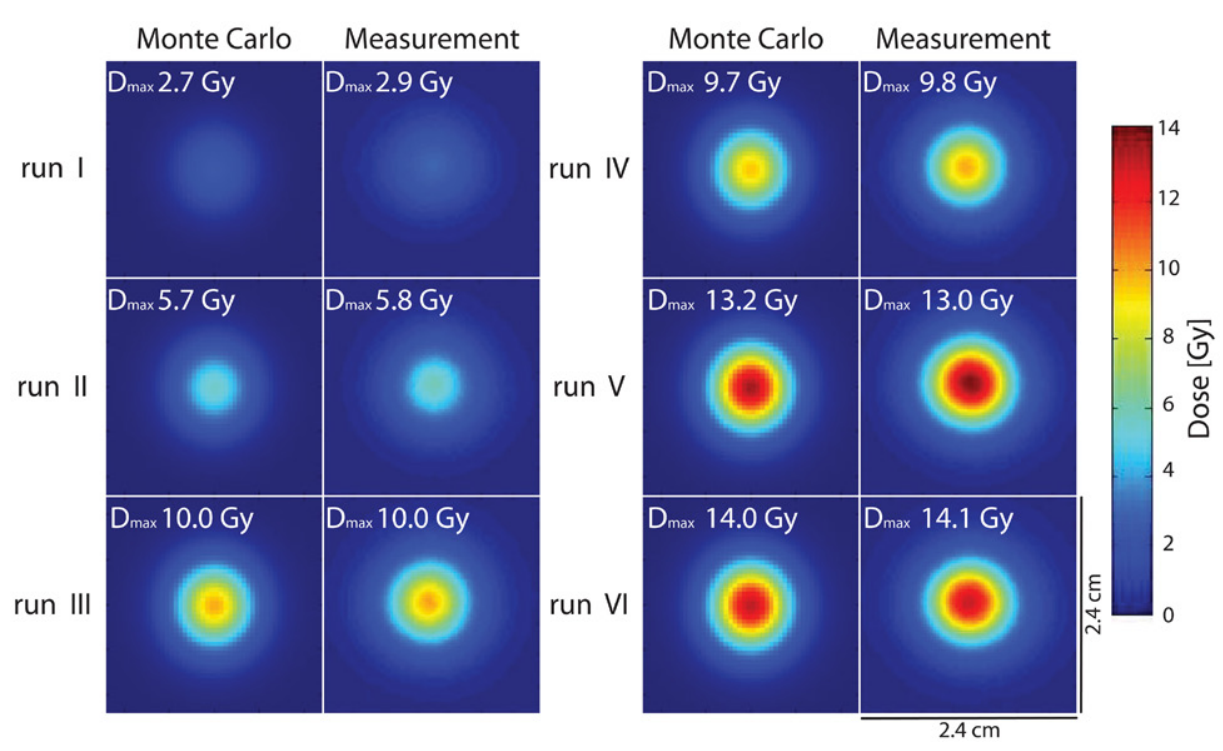

Figure 4. Measured and calculated dose maps of EBT2 films (lot\#: A08301204) irradiated with $165 \mathrm{MeV}$ electron beam.

uncertainty for the two EBT2 film batches within dose-levels for these studies using a calibrated $225 \mathrm{kV}$ x-ray source. For the investigated EBT2 film lot numbers the maximum difference relative to fitted dose is $6 \%$ and $10.1 \%$ for $10 \mathrm{cGy}$ and $100 \mathrm{cGy}$ dose levels, respectively.

The calibration curve from the experiment with $20 \mathrm{MeV}$ electrons is used below for the comparison of measurements with VHEE and the results of the Monte Carlo simulations.

\subsection{EBT2 film calibration with $165 \mathrm{MeV}$ electron beam}

The EBT2 films (lot\#: A08301204) irradiated with $165 \mathrm{MeV}$ SPARC electron beam have been analysed using the calibration curve shown in figure 3 . The measured and calculated values for $165 \mathrm{MeV}$ irradiation dose maps are shown in figure 4 , and the irradiation parameters with dose uncertainties are given in table 3 .

\subsection{Depth-dose measurements for VHEE}

The measured EBT2 film depth-dose profiles (obtained from the calibration curve shown in figure 3) along the central beam axis for the SPARC LINAC and ALPHA-X (LWFA) accelerators are given in figures 5 and 6 . The combined standard uncertainties of measured dose for both EBT2 lot numbers have been calculated for every dose level. The FLUKA simulated energy deposition curves representing absolute dose delivered are also shown in Gy. All dose measurements in these studies are calibrated using the calibration curve shown in figure 3 .

The lateral profiles have a strong dependence on initial beam divergence. The laser-plasma accelerated ALPHA-X electron beam has a quasi-Gaussian lateral profile at the entrance of the water phantom. This shape is due to multiple Coulomb scattering of the beam while propagating in air. Coulomb scattering in water leads to an increased lateral dose distribution area along the propagation direction and thus to a decreased peak dose measured by the Gafchromic ${ }^{\circledR}$ film sheets located in the water tank. 
Table 3. The summary of measured and calculated absorbed doses in EBT2 film on the beam central axis.

\begin{tabular}{llllrc}
\hline run \# & $\begin{array}{l}\text { \# of } \\
\text { shots }\end{array}$ & $\begin{array}{l}\text { charge/ } \\
\text { shot }[p C]\end{array}$ & $\begin{array}{l}\text { charge/shot } \\
S D[p C]\end{array}$ & $\begin{array}{l}\text { MC calculat- } \\
\text { ed dose }[G y]\end{array}$ & $\begin{array}{c}\text { Measured ab- } \\
\text { sorbed dose [Gy] }\end{array}$ \\
\hline run I & 11 & 56.5 & 3.2 & $2.7 \pm 0.1$ & $2.9 \pm 0.1$ \\
run II & 20 & 69.1 & 4.5 & $5.7 \pm 0.3$ & $5.8 \pm 0.2$ \\
run III & 38 & 64.3 & 4.4 & $10.0 \pm 0.6$ & $10.0 \pm 0.4$ \\
run IV & 41 & 57.7 & 2.4 & $9.7 \pm 0.3$ & $9.8 \pm 0.4$ \\
run V & 62 & 55.1 & 4.9 & $13.2 \pm 1.0$ & $13.0 \pm 0.7$ \\
run VI & 59 & 56.8 & 4.0 & $14.0 \pm 0.6$ & $14.1 \pm 0.7$ \\
\hline
\end{tabular}
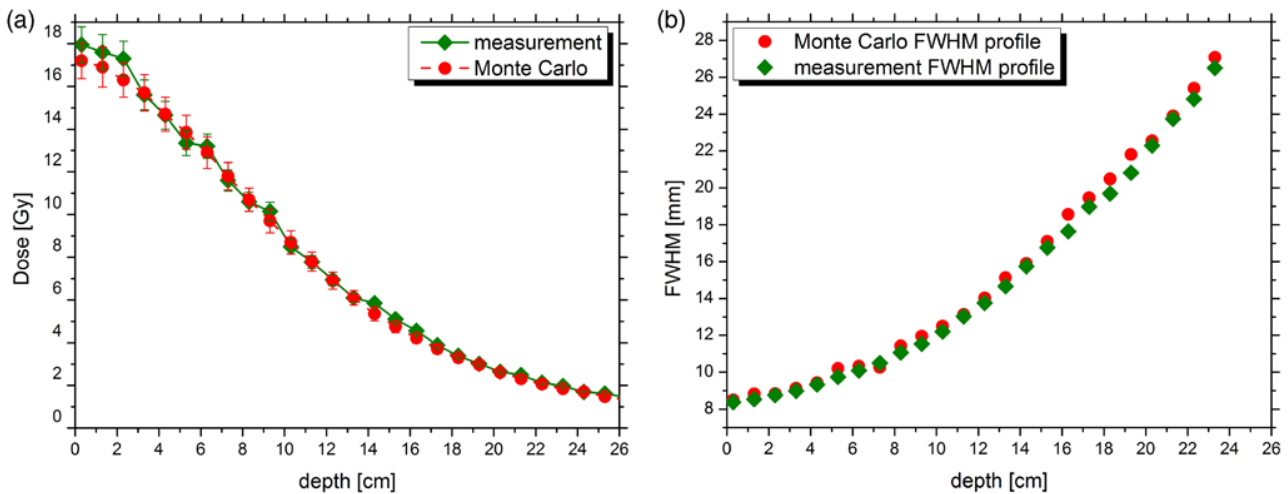

Figure 5. (a) Depth dose profile for $165 \mathrm{MeV}$ electron beam from the SPARC beamline. (b) Evolution of the FWHM beam profile as a function of depth within the water phantom.

\subsection{Monte Carlo simulations}

3.4.1. Depth dose characteristic. A set of Monte Carlo simulations in water have been performed to evaluate the dose distribution for VHEE beams generated by the LWFA. Both the ALPHA-X beamline and conventional high energy LINAC (SPARC) are simulated in this way. Results of these simulations are presented in figures 5 and 6. Ten million particles have been used for the calculations. The evaluated statistical uncertainty on the beam axis is below $1 \%$ up to $6 \mathrm{~cm}$ depth and reaches $2.5 \%$ at the depth of $20 \mathrm{~cm}$ for the ALPHA-X beam, while for the SPARC beam it is below $1 \%$ for the depth range of $0-20 \mathrm{~cm}$ and increases to $2 \%$ at $30 \mathrm{~cm}$ depth. The MC calculated dose uncertainties shown in figures 5(a) and $6(a)$ are dominated by the charge variation during irradiation.

3.4.2. Evaluation of neutron production. The neutron fluence (neutrons $\mathrm{cm}^{-2}$ ), shown in figure 7 , is of the order of $10^{-5}$ neutrons per incident electron $\left(\sim 10^{4}\right.$ neutrons $\left.\left(\mathrm{cm}^{-2} \mathrm{~Gy}^{-1}\right)\right)$ in the area of dose deposition and $10^{-7}\left(\sim 10^{2}\right.$ neutrons $\left(\mathrm{cm}^{-2} \mathrm{~Gy}^{-1}\right)$ at $10 \mathrm{~cm}$ from the back wall of the water phantom.

3.4.3. Activation. The activity has been scored for the first $(0.3 \mathrm{~cm}$ depth $)$, middle $(14.3 \mathrm{~cm}$ depth) and last film (29.3 cm depth) distribution within the water tank for each layer of EBT2 film separately. The results expressed in absolute values $(\mathrm{Bq})$ and percentage activity are presented in table 4, for Gafchromic ${ }^{\circledR}$ EBT2 films irradiated with the SPARC LINAC. The activation due to the generation of radionuclides has been evaluated for each film layer separately. 

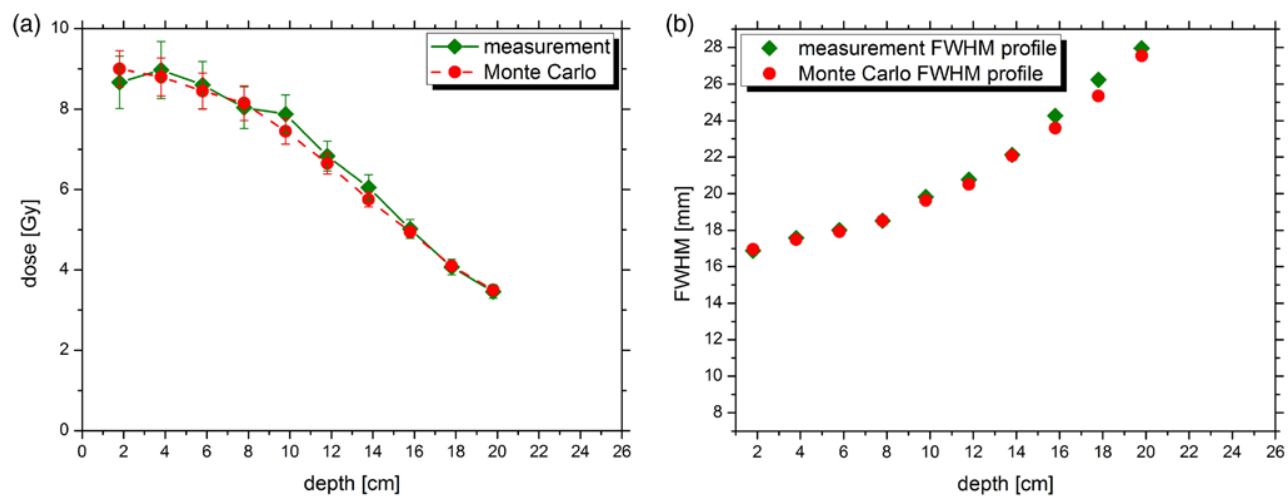

Figure 6. Depth dose profile for $135 \pm 44 \mathrm{MeV}$ (rms) laser-plasma accelerated electron beam in the ALPHA-X laboratory $(b)$ Evolution of the FWHM beam profile as a function of depth within water phantom.

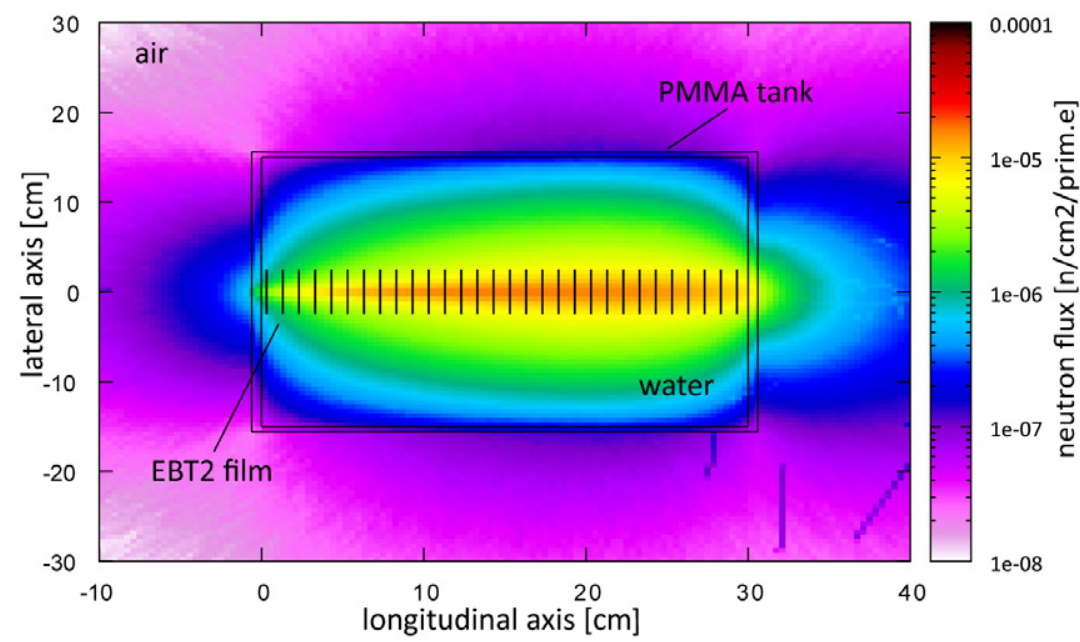

Figure 7. Neutron fluence inside and around the $30 \times 30 \times 30 \mathrm{~cm}^{3}$ water phantom with transversely distributed EBT2 films for $165 \mathrm{MeV}$ beams.

Activity in films at all depths for adhesive layer, topcoat and active layer $1 \mathrm{~min}$ after the beam irradiation ceased is determined by ${ }_{6}^{11} \mathrm{C}$ (22.3 min half-life) and ${ }_{8}^{15} \mathrm{O}$ (122.4 s half-life) radionuclides with the activity specified in table $4.20 \mathrm{~min}$ post-irradiation, the activity has been determined in these 3 layers of the film mostly by ${ }_{6}^{11} C$. In the protective polyester layers, activation of the ${ }_{6}^{11} \mathrm{C}$ radionuclide is significant.

In absolute terms, the total activity for the whole film irradiated in SPARC is $1.6 \mathrm{~Bq}, 16.5$ $\mathrm{Bq}$ and $19.5 \mathrm{~Bq}$, for the first $(0.3 \mathrm{~cm}$ depth) middle $(14.3 \mathrm{~cm}$ depth) and last $(29.3 \mathrm{~cm}$ depth $)$ film, respectively, $20 \mathrm{~min}$ post irradiation. In comparison, the total activity for the ALPHA-X beam in corresponding positions of the films in the water tank (at 1.8, 9.8 and $19.8 \mathrm{~cm}$ ) is 6.1 $\mathrm{Bq}, 22.7 \mathrm{~Bq}$ and $33.9 \mathrm{~Bq}$, respectively. The amount of the radionuclides scored in EBT2 films increases towards exit of the water tank where the majority of $(\gamma, n)$ reactions occur. The difference in total activity corresponds to the difference in the dose delivered and the field size of the beams in the SPARC and ALPHA-X experiments (see section 3.2). 


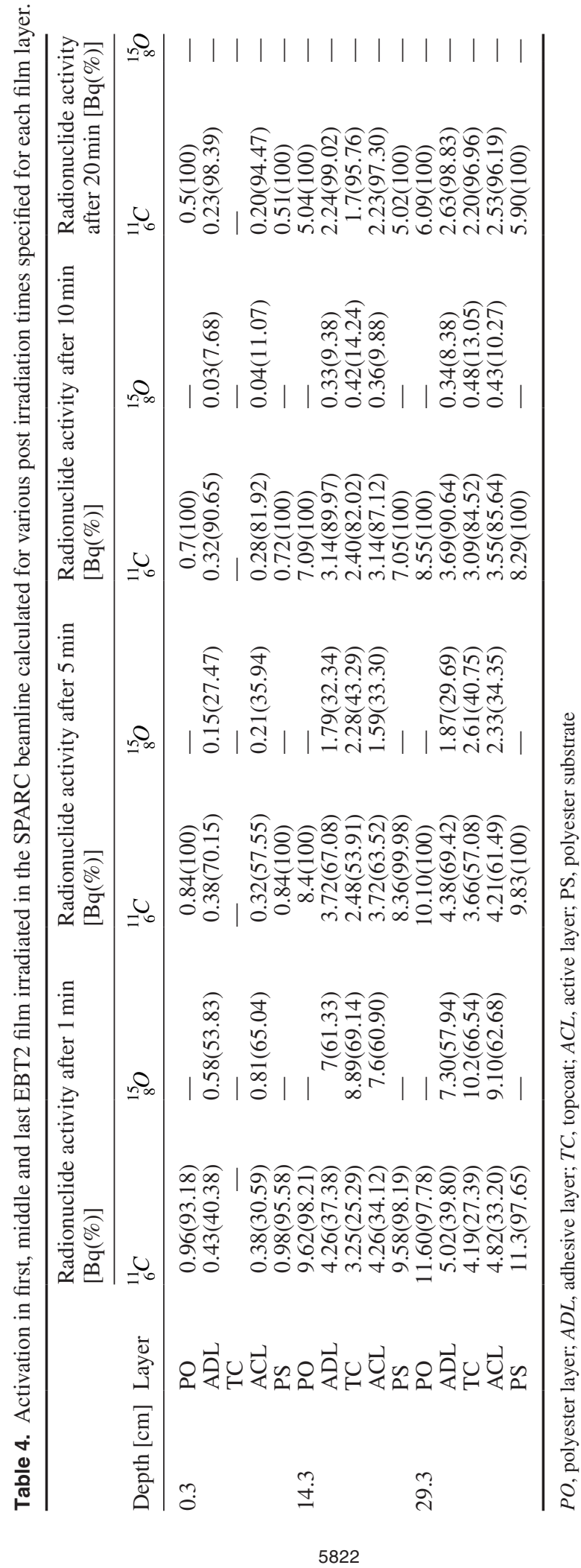


Table 5. Dose equivalent $\mathrm{H}^{*}(10)$ values calculated at $1 \mathrm{~mm}$ distance from the front and the rear wall of the water tank for various post-irradiation times for the SPARC beam.

\begin{tabular}{lccccc}
\hline & \multicolumn{2}{c}{ Front surface } & & \multicolumn{2}{c}{ Rear surface } \\
\cline { 2 - 3 } Post-irradiation time & $\mathrm{H}^{*}(10)$ rate $^{*}\left[\mathrm{pSv} \mathrm{s}^{-1}\right]$ & stat. error [\%] & & $\mathrm{H}^{*}(10)$ rate $\left[\mathrm{pSv} \mathrm{s}^{-1}\right]$ & stat. error [\%] \\
\hline $1 \mathrm{~min}$ & 125.31 & 0.53 & & 571.56 & 0.56 \\
$5 \mathrm{~min}$ & 36.70 & 0.48 & & 175.74 & 0.45 \\
$10 \mathrm{~min}$ & 11.46 & 0.45 & 61.04 & 0.24 \\
$15 \mathrm{~min}$ & 6.07 & 0.57 & & 35.37 & 0.20 \\
$20 \mathrm{~min}$ & 4.42 & 0.64 & 26.71 & 0.23 \\
\hline
\end{tabular}

Table 6. Dose equivalent $\mathrm{H}^{*}(10)$ values calculated at $1 \mathrm{~mm}$ distance from the front and the rear wall of the water tank for various post-irradiation times for the ALPHA-X beam.

\begin{tabular}{llllll}
\hline & \multicolumn{2}{c}{ Front surface } & \multicolumn{2}{c}{ Rear surface } \\
\cline { 2 - 3 } Post-irradiation time & $\mathrm{H}^{*}(10)$ rate $\left[\mathrm{pSv} \mathrm{s}^{-1}\right]$ & stat. error [\%] & & $\mathrm{H}^{*}(10)$ rate $\left[\mathrm{pSv} \mathrm{s}^{-1}\right]$ & stat. error [\%] \\
\hline $1 \mathrm{~min}$ & 69.41 & 0.34 & 345.14 & 0.18 \\
$5 \mathrm{~min}$ & 25.04 & 0.23 & 144.61 & 0.22 \\
$10 \mathrm{~min}$ & 11.34 & 0.12 & 78.79 & 0.30 \\
$15 \mathrm{~min}$ & 1.47 & 0.20 & 56.49 & 0.11 \\
$20 \mathrm{~min}$ & 0.73 & 2.40 & 47.39 & 0.36 \\
\hline
\end{tabular}

3.4.4. Equivalent dose. To confirm that activated doses do not present any safety concerns, the equivalent doses due to induced radioactivity have been calculated for the experimental conditions for the SPARC and ALPHA-X beamlines, separately. Tables 5 and 6 present the values of $\mathrm{H}^{*}(10)$ dose equivalent at $1 \mathrm{~mm}$ from the front and rear wall of the water tank surface for various post irradiation times during the dosimetry study in the SPARC and ALPHA-X beam lines, respectively. The post-irradiation time is set to a maximum of $20 \mathrm{~min}$, such that equivalent dose rates are significantly less than natural background levels $\left(\sim 100 \mathrm{pSv} \mathrm{s}^{-1}\right)$, after which the films are removed from the water.

20 min after irradiation the $\mathrm{H}^{*}(10)$ values are at the level of 26 and $47 \mathrm{pSv} \mathrm{s}^{-1}$ for the SPARC and ALPHA-X beams, respectively.

3.4.5. LET spectrum. The LET spectrum for the ALPHA-X beam (figure 8) has been scored using the FLUKA USRYIELD utility.

The LET spectra for the ALPHA-X beam have a maximum at $0.39 \mathrm{keV}_{\mu} \mathrm{m}^{-1}$, and a long tail

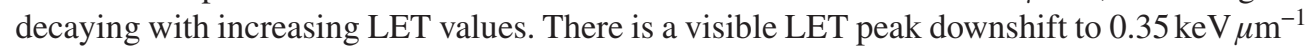
for the $20 \mathrm{MeV}$ electron beam.

\section{Discussion}

Previous Monte Carlo studies (DesRosiers et al 2000, Yeboah et al 2002, Moskvin et al 2010) came to the conclusion that VHEE beams have favourable penetrating properties and can achieve a very good dose conformation, comparable with or even exceeding the properties of current photon modalities. However, until now no absolute dosimetric evaluation for VHEEs has been available. Our studies present a reliable method of performing dose measurements for very high energy electron beams. 


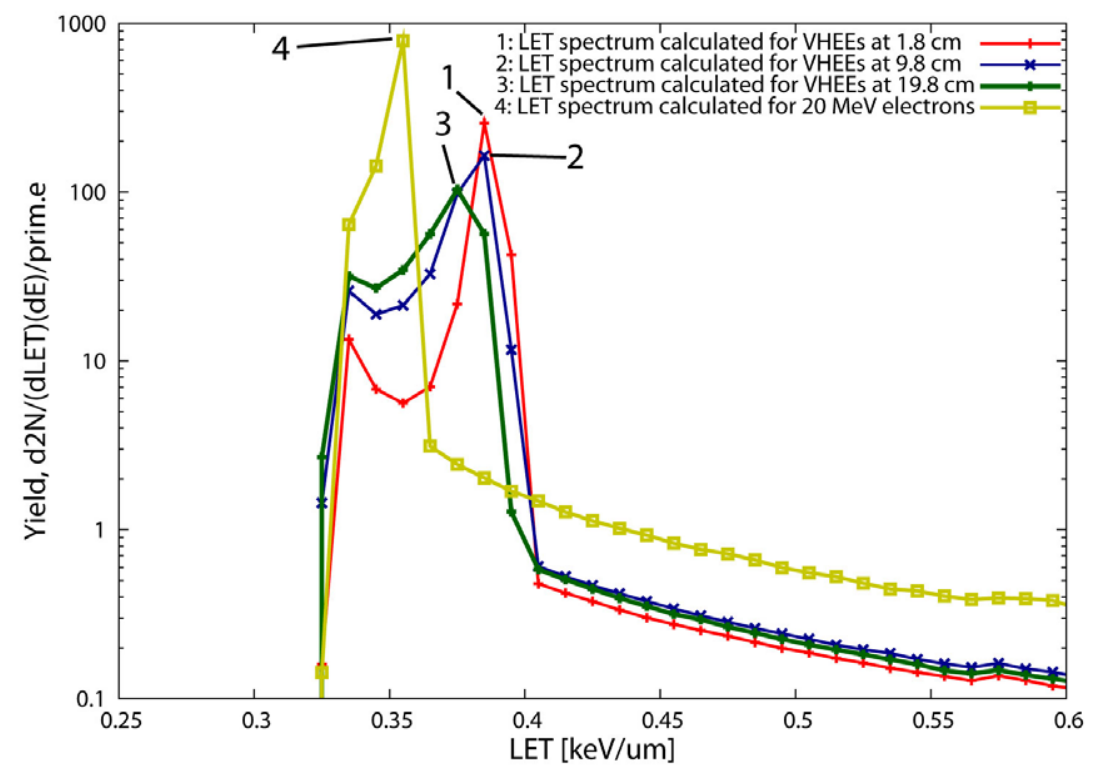

Figure 8. LET spectra calculated for $135 \mathrm{MeV}$ ALPHA-X electron beams at EBT2 films positioned at $1.8,9.8$ and $19.8 \mathrm{~cm}$ in the water bath. The calculated $20 \mathrm{MeV}$ electron LET spectrum is included for comparison.

The measured dose maps of EBT2 calibration films using the $165 \mathrm{MeV}$ electron beam, shown in figure 4, agree well with Monte Carlo calculations. The main uncertainties in simulations arise from charge/shot jitter, which has been quantified in table 3 for the dose deposited by the SPARC VHEEs at the beam central axis.

The measured depth dose profiles along the central axis of the beam (established in absolute terms) with transversely orientated EBT2 films in the water phantom for the $165 \mathrm{MeV}$ LINAC (figure 5(a)) and $135 \mathrm{MeV}$ laser-plasma generated (figure 6(a)) beams are in excellent agreement with the Monte Carlo calculations. The difference in depth dose profiles between VHEE LINAC and LWFA electrons result from their distinct beam parameters i.e. primary energy, divergence and lateral beam size. For $165 \mathrm{MeV}$ electrons from the SPARC beamline, the beam emerging from the water tank has a field size of $0.8 \mathrm{~cm}$ FWHM with a $15 \mathrm{mrad}$ divergence. In the case of ALPHA-X electrons the beam field size is twice as large: $1.6 \mathrm{~cm}$ FWHM and a divergence of $8 \mathrm{mrad}$. Therefore the practical range $\left(R_{\mathrm{p}}\right)$ for the ALPHA-X beam exceeds that of the SPARC beam. It is well known that with a reduction of field size there is a lower level of lateral electronic equilibrium at the beam central axis. As a consequence, the depth dose should show high sensitivity to field shape and size (Podgorsak 2005). The clinical beams used in dosimetry of radiotherapy LINACs have a field size of at least $10 \mathrm{~cm} \times 10 \mathrm{~cm}$ to achieve scatter equilibrium. A clinical LINAC electron beam is uniform and broad because of the scattering foils that are commonly used. When the field size is reduced significantly, as in the case of the VHEE beams studied, electrons from the periphery of the field are not scattered sufficiently to contribute to the central axis depth dose, and therefore $D_{\max }$ moves closer to the entrance (front wall) of the water phantom, which is obvious in figures 5(a) and 6(a). This effect has also been observed by (Lundh et al 2012) for a LWFA electron beam with a diameter of approximately $0.2 \mathrm{~cm}$. However, this work did not include any absolute dose measurements because the detector used in the studies, image plates (IP), are not designed to measure absorbed dose. In clinical applications, 
VHEE beams can be scanned using electromagnetic magnets to deliver intensity-modulated radiation treatment (Papiez et al 2004). Since this method of the beam delivery will not involve the interaction of the electron beam with any type of the flattening filter, the neutron contamination from the machine head is minimized.

The evolution of FWHM beam lateral profiles at various depths in the water phantom for SPARC (figure 5(b)) and ALPHA-X (figure 6(b)) strongly depend on the initial beam divergence and even though the initial beams for the two are within a factor of 2 in size, the FWHM field size close to the rear of the water phantom for the two cases is approximately the same due to multiple Coulomb scattering which deflects electrons transversely. The beam profile at the rear of the water phantom has a much larger diameter than that of the initial profile.

For VHEEs, there are two regions of photon energies with respect to neutron production mechanisms, i.e. the giant dipole resonance (GDR) giving rise to a $(\gamma, n)$ reaction threshold that is approximately equal to the binding energy of the nucleon (for photon energies between the threshold and $40 \mathrm{MeV}$ ), and processes above the giant resonance are more important for high $\mathrm{Z}$ number materials. The neutron yield also increases with the bremsstrahlung contribution. This is pronounced in figure 7 , which shows the quasi-isotropic neutron fluence around and inside the water phantom. As the VHEE beam propagates through water the mean and peak electron energy decreases while the bremsstrahlung photon flux increases with increasing depth. The isotropic nature of the neutron emission is due to the dominance of the GDR mechanism (Mao et al 1996), where the emitted neutrons are due to evaporation neutrons from a compound nucleus. The deviation from ideally isotropic behaviour for VHEEs is a consequence of anisotropic emission of neutrons from other processes such as quasi-deuteron effects where photons interact with the neutron-proton pair within the nucleus, rather than the nucleus as a whole. These studies involve the estimation of neutron flux generated in water that contributes to the dose delivered inside the phantom. The Monte Carlo calculated neutron fluence inside the phantom for $165 \mathrm{MeV}$ VHEEs is of the order $10^{-5}$ neutrons/ $\left(\mathrm{cm}^{2}\right.$.primary electrons). This value is almost three orders of magnitude lower than the results for $150 \mathrm{MeV}$ electrons presented in previous studies based on a semi-empirical evaluation (DesRosiers et al 2000) established for a higher particle beam density approach. According to Howell et al (Howell et al 2009) the neutron fluence due to secondary neutron emission in the Varian $21 \mathrm{EX}$, for $20 \mathrm{MV}$ nominal x-ray energy at the depth of the maximum dose for $10 \times 10 \mathrm{~cm}^{2}$ field, is $1.69 \cdot 10^{5}$ neutrons $\left(\mathrm{cm}^{-2} \mathrm{MU}^{-1}\right)$ This value is one order of magnitude larger than the neutron fluence calculated inside a water phantom irradiated with VHEEs. The total activity of the films due to radioisotope generation in both SPARC and ALPHA-X beamlines is of the order of $10 \mathrm{~Bq}$, depending on the film position inside the water tank (details on induced activity for the SPARC beam in table 4). The induced activity increases slightly with increasing depth due to the accumulation of bremsstrahlung generation downstream where the majority of $(\gamma, n)$ reactions occur. The dose equivalent rates due to low induced radioactivity of ${ }_{6}^{11} \mathrm{C}$ and ${ }_{8}^{15} \mathrm{O}$ play a minor role here. After $20 \mathrm{~min}$ of post irradiation time, which corresponds to the moment of removing the films from the water phantom, the dose equivalent rate is of the order of $20-50 \mathrm{pSv} \mathrm{s}^{-1}$ on the beam central axis at the rear of the water phantom. Thus we can conclude that neutron and proton production due to irradiation of VHEEs does not significantly affect the equivalent doses. In summary, the induced radioactivity from neutron fluence is found to be small and therefore it has negligible effect on total dose deposition.

The investigated LWFA VHEE beams have characteristics unlike conventional radiotherapy beams. Relativistic effects of these beams resulting of their high energies and ultra-short duration (few fs) in the dose delivery could cause the different response to irradiation in tissue 
(Malka et al 2010) compared with conventional LINAC produced electrons and photons. These features could make a VHEE beam an interesting candidate as a new modality in radiotherapy, with advantages over existing methods. It is therefore important to compare VHEEs with other radiotherapy sources, including ions.

There have been several investigations considering radiochromic films as a dosimetric tool in proton therapy (Kirby et al 2010, Zhao and Das 2010, Angellier et al 2011). These studies show an under-response of EBT film at the Bragg peak, which suggest a film response dependence on LET. However, in our work we have not observed any difference in film response for low and very high energy electron beams. The LET spectra shown in figure 8 , has been calculated using a MC method. When comparing the calculated LET spectra for $20 \mathrm{MeV}$ and VHEE beams one notices an upshift of the LET peak for LWFA VHEEs, however this difference is small. Because the LET spectra for both beams, low energy electrons and VHEEs, are very similar one could expect the EBT2 film response difference to be indistinguishable for these modalities.

\section{Conclusions}

The results of this study demonstrate that the EBT2 Gafchromic film is a reliable dosimeter for ultra-short pulses of very high energy electron beams generated by laser-plasma accelerators. Our study indicates energy-independence of EBT2 Gafchromic film over the investigated electron energies. The dosimetry with EBT2 Gafchromic film assures measurement of the dose for the non-equilibrium, small field of very high energy electrons within 3.5-5.4\% of experimental uncertainty. Monte Carlo simulations with the FLUKA generalpurpose code confirm the EBT2 Gafchromic film method of dosimetry and demonstrate the usefulness of the code for interpreting experimental studies using very high energy electron beams in the range of $130-170 \mathrm{MeV}$. It has also been calculated that the neutron yield is lower than predicted in early studies of VHEE (DesRosiers et al 2000). EBT2 dosimetry will underpin any further work aiming to demonstrate versatility of potential application of VHEEs to radiotherapy.

\section{Acknowledgements}

This work has been supported by EPSRC grant EP/J018171/1, STFC grants ST/ H003819/1, ST/H003703/1 and ST/H003754/1, Clarian Values Grant VFR-273 and CSO grant ETM/194. Thanks are extended to John Frame from the Beatson Oncology Centre for valuable advice regarding EBT film dosimetry and the Radiotherapy Department team at the Royal Surrey County Hospital (in particular to Tom Jordan and Matthew Jones) who helped with $20 \mathrm{MeV}$ electron calibration of EBT2 films used in these studies. We would also like to acknowledge David Clark and Thomas McCanny at Strathclyde for their valuable technical support.

\section{References}

Alesini D et al 2003 The SPARC project: a high-brightness electron beam source at LNF to drive a SASE-FEL experiment Nucl. Instrum. Methods Phys. Res. A 507 345-9

Ali I, Costescu C, Vicic M, Dempsey J and Williamson J 2002 Dependence of radiochromic film optical density post-exposure darkening kinetics on dose and dose fractionation Med. Phys. 291351 
Andres C, del Castillo A, Tortosa R, Alonso D and Barquero R 2010 A comprehensive study of the Gafchromic EBT2 radiochromic film. A comparison with EBT Med. Phys. 37 6271-8

Angellier G, Gautier M and Herault J 2011 Radiochromic EBT2 film dosimetry for low-energy protontherapy Med. Phys. 38 6171-7

Arjomandy B, Tailor R, Anand A, Sahoo N, Gillin M, Prado K and Vicic M 2010 Energy dependence and dose response of Gafchromic EBT2 film over a wide range of photon, electron, and proton beam energies Med. Phys. 37 1942-7

Arjomandy B, Tailor R, Zhao L and Devic S 2012 EBT2 film as a depth-dose measurement tool for radiotherapy beams over a wide range of energies and modalities Med. Phys. 39 912-21

Ashland 2013a (www.filmqapro.com/Documents/GafChromic_EBT-2_20101007.pdf)

Ashland 2013b (www.gafchromic.com)

Barbier M M 1969 Induced Radioactivity (New York: North-Holland)

Battistoni G, Muraro S, Sala P R, Cerutti F, Ferrari A, Roesler S, Fasso A and Ranft J 2007 The FLUKA code: description and benchmarking Proc. of the Hadronic Shower Simulation Workshop, Fermilab 6-8 September 2006 ed M Albrow and R Raja vol 896 (Melville, NY: AIP Conference Proceedings) pp 31-49

Bouchard H, Lacroix F, Beaudoin G, Carrier J-F and Kawrakow I 2009 On the characterization and uncertainty analysis of radiochromic film dosimetry Med. Phys. 36 1931-46

Brunetti E et al 2010 Low emittance, high brilliance relativistic electron beams from a laser-plasma accelerator Phys. Rev. Lett. 1054

Butson M J, Yu P K N, Cheung T and Alnawaf H 2010 Energy response of the new EBT2 radiochromic film to x-ray radiation Radiat. Meas. 45 836-9

Chung H T, Lynch B and Samant S 2010 High-precision GAFCHROMIC EBT film-based absolute clinical dosimetry using a standard flatbed scanner without the use of a scanner non-uniformity correction J. Appl. Clin. Med. Phys. 11 101-15

Das I J, Ding G X and Ahnesjo A 2008 Small fields: nonequilibrium radiation dosimetry Med. Phys. 35 206-15

DesRosiers C, Moskvin V, Bielajew A F and Papiez L 2000 150-250 MeV electron beams in radiation therapy Phys. Med. Biol. 45 1781-805

DesRosiers C, Moskvin V, Cao M, Joshi C and Langer M 2008a Lung tumor treatment with very high energy electron beams of 150-250 Mev as compared to conventional megavoltage photon beams Int. J. Radiat. Oncol. Biol. Phys. 72 S612

DesRosiers C, Moskvin V, Cao M, Joshi C J and Langer M 2008b Laser-plasma generated very high energy electrons in radiation therapy of the prostate Proc. SPIE 6881688109

Devic S 2011 Radiochromic film dosimetry: past, present, and future Phys. Med.-Eur. J. Med. Phys. 27 122-34

Devic S, Seuntjens J, Hegyi G, Podgorsak E. B, Soares C G, Kirov A S, Ali I, Williamson J F and Elizondo A 2004 Dosimetric properties of improved GafChromic films for seven different digitizers Med. Phys. 31 2392-401

Faure J, Glinec Y, Pukhov A, Kiselev S, Gordienko S, Lefebvre E, Rousseau J P, Burgy F and Malka V 2004 A laser-plasma accelerator producing monoenergetic electron beams Nature 431 541-4

Ferrari A, Sala P R, Fasso A and Ranft J 2005 FLUKA: A Multi-Particle Transport Code CERN 2005-10, INFN/TC_05/11, SLAC-R-773

Fuchs T, Szymanowski H, Oelfke U, Glinec Y, Rechatin C, Faure J and Malka V 2009 Treatment planning for laser-accelerated very-high energy electrons Phys. Med. Biol. 54 3315-28

Geddes C G R, Toth C, van Tilborg J, Esarey E, Schroeder C B, Bruhwiler D, Nieter C, Cary J and Leemans W P 2004 High-quality electron beams from a laser wakefield accelerator using plasmachannel guiding Nature $\mathbf{4 3 1} 538-41$

Glinec Y, Faure J, Malka V, Fuchs T, Szymanowski H and Oelfke U 2006 Radiotherapy with laser-plasma accelerators: Monte Carlo simulation of dose deposited by an experimental quasimonoenergetic electron beam Med. Phys. 33 155-62

Hogstrom K R and Almond P R 2006 Review of electron beam therapy physics Phy. Med. Biol. 51 R455-89

Howell R M, Kry S F, Burgett E, Hertel N E and Followill D S 2009 Secondary neutron spectra from modern Varian, Siemens, and Elekta linacs with multileaf collimators Med. Phys. 36 4027-38

IAEA 1979 Radiobiological Safety Aspects of the Oparation of Electron Linear Accelerators (Vienna: International Atomic Energy Agency) 
ICRP 1996 Conversion Coefficients for Use in Radiological Protection Against External Radiation ICRP Publication 74 (Oxford: Pergamon)

Jaroszynski D A et al 2000 The strathclyde terahertz to optical pulse source (TOPS) Nucl. Instrum. Methods Phys. Res. A 445 317-9

JCGM 2008 Evaluation of measurement data-guide to the expression of uncertainty in measurement Joint Committee for Guides in Metrol. 100

Kirby D, Green S, Palmans H, Hugtenburg R, Wojnecki C and Parker D 2010 LET dependence of GafChromic films and an ion chamber in low-energy proton dosimetry Phys. Med. Biol. 55 417-33

KODAK (www.carestream.com/lanex-screens.html)

Lindsay P, Rink A, Ruschin M and Jaffray D 2010 Investigation of energy dependence of EBT and EBT-2 Gafchromic film Med. Phys. 37 571-6

Lomax A J, Bortfeld T, Goitein G, Debus J, Dykstra C, Tercier P A, Coucke P A and Mirimanoff R O 1999 A treatment planning inter-comparison of proton and intensity modulated photon radiotherapy Radiother. Oncol. 51 257-71

Lundh $\mathrm{O}$ et al 2011 Few femtosecond, few kiloampere electron bunch produced by a laser-plasma accelerator Nat. Phys. 7 219-22

Lundh O, Rechatin C, Faure J, Ben-Ismail A, Lim J, De Wagter C, De Neve W and Malka V 2012 Comparison of measured with calculated dose distribution from a $120 \mathrm{MeV}$ electron beam from a laser-plasma accelerator Med. Phys. 39 3501-8

Malka V, Faure J and Gauduel Y A 2010 Ultra-short electron beams based spatio-temporal radiation biology and radiotherapy Mutat. Res.-Rev. Mutat. Res. 704 142-51

Mangles S P D et al 2004 Monoenergetic beams of relativistic electrons from intense laser-plasma interactions Nature $\mathbf{4 3 1} 535-8$

Mao X T, Kase K R and Nelson W R 1996 Giant diople resonance neutron yields produced by electrons as a function of target material and thickness Health Phys 70 207-14

Moskvin V, Salvat F, Stewart D K and DesRosiers C M 2010 PENELOPE Monte Carlo engine for treatment planning in radiation therapy with very high energy electrons (VHEE) of 150-250 MeV IEEE Nuclear Science Symp. Conf. Record (NSS/MIC) 1961-66

NCRP 2003 Radiation protection for particle accelerator facilities NCRP Report No. 144 (Bethesda, MD: NCRP)

Niroomand-Rad A, Blackwell C R, Coursey B M, Gall K P, Galvin J M, McLaughlin W L, Meigooni A S, Nath R, Rodgers J E and Soares C G 1998 Radiochromic film dosimetry: recommendations of AAPM Radiation Therapy Committee Task Group 55 Med. Phys. 25 2093-115

Paelinck L, Ebongue A N, De Neve W and De Wagter S 2007 Radiochromic EBT film dosimetry: effect of film orientation and batch on the lateral correction of the scanner Radiother. Oncol. 84 S194-5

Palmer D T 1998 The next generation photoinjector PhD Thesis Stanford University, California

Papiez L, Bortfeld T and Hendee W R 2004 Very high energy electromagnetically-scanned electron beams are an attractive alternative to photon IMRT Med. Phys. 31 1945-8

Parodi K, Paganetti H, Cascio E, Flanz J B, Bonab A A, Alpert N M, Lohmann K and Bortfeld T 2007 PET/CT imaging for treatment verification after proton therapy: a study with plastic phantoms and metallic implants Med. Phys. 34 419-35

Paterson I J, Clarke R J, Woolsey N C and Gregori G 2008 Image plate response for conditions relevant to laser-plasma interaction experiments Meas. Sci. Technol. 19095301

Pelliccioni M 2000 Overview of fluence-to-effective dose and fluence-to-ambient dose equivalent conversion coefficients for high energy radiation calculated using the FLUKA code Radiat. Prot. Dosim. 88 279-97

Podgorsak E B 2005 Radiation Oncology Physics (Vienna: International Atomic Energy Agency)

Randeniya S D, Taddei P J, Newhauser W D and Yepes P 2009 Intercomparision of Monte Carlo radiation transport codes MCNPX, GEANT4, and FLUKA for simulating proton radiotherapy of the eye Nucl. Technol. 168 810-4

Reinhardt S, Hillbrand M, Wilkens J J and Assmann W 2012 Comparison of Gafchromic EBT2 and EBT3 films for clinical photon and proton beams Med. Phys. 39 5257-62

Richter C, Kaluza M, Karsch L, Schlenvoigt H P, Schurer M, Sobiella M, Woithe J and Pawelke J 2011 Dosimetry of laser-accelerated electron beams used for in vitro cell irradiation experiments Radiat. Meas. 46 2006-9

Robert C et al 2013 Distributions of secondary particles in proton and carbon-ion therapy: a comparison between GATE/Geant4 and FLUKA Monte Carlo codes Phys. Med. Biol. 58 2879-99 
Shanks R P 2012 Comprehensive Characterisation of Laser Plasma Wakefield Accelerated Electrons (Glasgow: University of Strathclyde)

SPARC (www.lnf.infn.it/acceleratori/sparc/)

Sutherland J G H and Rogers D W O 2010 Monte Carlo calculated absorbed-dose energy dependence of EBT and EBT2 film Med. Phys. 37 1110-6

Swanson W P 1978 Calculation of neutron yields released by electrons incident on selected materials Health Phys. 353-67

Varian (www.varian.com/us/oncology/radiation_oncology/clinac/clinac_ix.html)

Wiggins S M et al 2010 High quality electron beams from a laser wakefield accelerator Plasma Phys. Control. Fusion $\mathbf{5 2} 124032$

Yeboah C and Sandison G A 2002 Optimized treatment planning for prostate cancer comparing IMPT, VHEET and 15 MV IMXT Phys. Med. Biol. 47 2247-61

Yeboah C, Sandison G A and Moskvin V 2002 Optimization of intensity-modulated very high energy (50-250 MeV) electron therapy Phys. Med. Biol. 47 1285-301

Zhao L and Das I I 2010 Gafchromic EBT film dosimetry in proton beams Phys. Med. Biol. 55 